\title{
Stochastic Petri Nets in Systems Biology Ole Schulz-Trieglaff*
}

\author{
Address: School of Informatics, University of Edinburgh, Edinburgh EH9 3JZ, UK. \\ Email: Ole Schulz-Trieglaff* - O.schulz-trieglaf@sms.ed.ac.uk \\ * Corresponding author
}

from BioSysBio: Bioinformatics and Systems Biology Conference

Edinburgh, UK, 14-15 July 2005

Published: 2I September 2005

BMC Bioinformatics 2005, 6(Suppl 3):P25

Stochastic Petri Nets are a well-known class of models that has been extensively used by computer scientists to analyse distributed and parallel systems. The Petri Net theory as it was developed by Adam Petri [5] offer means to analyse topological properties of the net such as the existence of deadlocks or invariants. With their stochastic extension, Petri Nets can also be used to model the dynamics of stochastic systems. The underlying Markov Process and information about its steady state behavior can easily be obtained.

All these properties have proven to be useful in the context of biological applications [6]. For instance some topological properties of the net are also meaningful in a biological con-text [7]. In addition, Stochastic Petri Nets have also been successfully applied to simulate random fluctuations on the level of gene expression [1]. In contrast to other modelling tools for biological systems, Petri Nets offer a more formal view and the underlying theory is well understood.

Nevertheless there are no editors for Stochastic Petri Nets that are tailored for biological applications. As an example, the Systems Biology Markup Language [3] is a standard for the storage of biological models, which allows exchanging models between different programs. But so far there is no Petri Net tool that supports this markup language.

For these reasons I develop a Stochastic Petri Net module for the Systems Biology Workbench (SBW) [2]. The module is based on the Open Source project The Petri Net Kernel [4]. This project is a framework for the editing and simulation of Petri Nets. I implemented support for Stochastic Nets and SBML as well as an interface to the Systems Biology Workbench. Using this interface various simulation features will be available. I will validate this software with a simple Petri Net model of Volterra-Lotka reactions and its stochastic simulation. In the near future I will use this software to further explore issues that arise from the application of Stochastic Petri Nets to biological systems.

There are several open questions that would be worthwhile to address. For instance, can the topology of the net be used to compute its steady state distribution more efficiently? Other interesting points are the representation of membranes, diffusion processes or reversible reactions in the Petri Net. The software will be made available online.

\section{References}

I. Goss PJE, Peccoud J: Quantitative modelling of stochastic systems in molecular biology by using stochastic Petri nets. Proc Natl Acad Sci USA 1998, 95:6750-6755.

2. Hucka M, Finney A, Sauro HM, Bolouri H, Doyle J, Kitano H: The ERATO Systems Biology Workbench: Enabling Interaction and Exchange between Software Tools for Computational Biology. Proceedings of the Pacific Symposium on Biocomputing 2002.

3. Hucka M, et al.: The Systems Biology Markup Language (SBML): A medium for representation and exchange of biochemical network models. Bioinformatics 2003, 19(4):524-531.

4. Kindler E, Weber M: The Petri Net Kernel - An Infrastructure for Building Petri Net Tools. Software Tools for Technology Transfer (STTT) 200I, 3(4):486-497.

5. Petri CA: Kommunikation mit Automaten Institut fur Instrumentelle Mathematik Bonn. Schriften des IIM 1962, 3:.

6. Reddy VN, Mavrovouniotis ML, Liebman MN: Petri net representations in metabolic pathways. Proceedings of the ISMB 1993:328-336.

7. Zevedei-Oancea I, Schuster S: Topological analysis of metabolic networks based on Petri net theory. Silico Biology 2003, 3:0029. 